\title{
EFFECTS OF ELECTRODE CONFIGURATION AND GEOMETRY ON FIBER PREFERENCE IN SPINAL CORD STIMULATION
}

\author{
Jan Holsheimer, Johannes J. Struijk* , Wilbert A. Wesselink \\ Institute for Biomedical Technology, University of Twente, P.O. Box 217, \\ 7500 AE Enschede, The Netherlands. e-mail: j.holsheimer@el.utwente.nl
}

\begin{abstract}
In contrast to the widespread assumption that dorsal column fibers are the primary targets of spinal cord stimulation by a dorsal epidural electrode, it appears that dorsal root fibers are recruited as well, and even preferentially under various conditions. This will, however, limit the coverage of the painfull body areas with paresthesia, a prerequisite for the management of chronic pain. In order to favor the preferential stimulation of dorsal column fibers, advantage was taken of the different positions and orientations of fibers in the dorsal columns and dorsal roots. Using an SCS computer model, electrode configurations have been designed for the selective stimulation of the human dorsal columns.
\end{abstract}

\section{INTRODUCTION}

It has long been assumed that in spinal cord stimulation (SCS) for the management of chronic, intractable pain the large nerve fibers in the dorsal columns of the spinal cord are the primary targets when stimulating by a dorsal epidural electrode. However, computer modeling has shown that large dorsal root (DR) fibers almost need the same stimulus strength for their excitation as dorsal column (DC) fibers and, under some conditions, even less [1]. The main factors causing this low DR fiber threshold are the fiber orientation in the electric field imposed by a longitudinal anode-cathode configuration, the transition from the well-conducting cerebrospinal fluid to the lowconducting dorsal columns, and the caliber and curvature of the DR fibers. At a stimulus strength of approx. $40 \%$ above a patient's first perception of paresthesia, SCS results in discomfortable motor effects, presumably related to the stimulation of smaller DR fibers. The resulting small therapeutic amplitude window limits the stimulation of DC fibers. The stimulation of large fibers in just a few dorsal rootlets and in only part of the dorsal columns often prevents the necessary coverage of the painful dermatomes by paresthesia, especially in complex pain patterns. Clinical data are generally in accordance with these model predictions.

* Present adress: University of Aalborg, Dept. of Medical Informatics \& Image Analysis, Frederik Bajers vej $7 D$, 9220 Aalborg,Denmark. e-mail: jjs@miba.auc.dk
The theoretical analysis has shown that improving the performance of SCS by increasing paresthesia coverage should be realized by raising the number of $D C$ fibers recruited within the therapeutic window and/or reducing the activation of DR fibers. Since the orientations of both fiber types are almost perpendicular and the dorsal roots are at the lateral sides of the dorsal columns, these effects might be obtained by applying current by different anodecathode combinations and geometries, thereby varying the imposed electric field. The UT-SCS computer model has been used to model various electrode configurations and to simulate their effects on dorsal column fiber and dorsal root fiber thresholds.

\section{METHODS}

The UT-SCS computer model consists of two parts. The first part is a $3 \mathrm{D}$ volume conductor model, representing the geometry and electrical conductivities of a $60 \mathrm{~mm}$ long segment of the spinal cord, its surrounding anatomical structures (cerebrospinal fluid, dura mater, epidural fat, vertebral bone) and a border layer representing distant tissues. A volume conductor model consists of 175,616 cubic volume elements of variable dimensions. Anodal and cathodal contacts are placed in the dorsal epidural space next to the dura mater and are set at different voltages. The voltages at the grid points of the $3 \mathrm{D}$ model are calculated by applying a finite difference method to discretize the governing Laplace equation. The resulting set of linear equations is solved using a Red-Black GaussSeidel iteration with variable overrelaxation [2].

The second part of the SCS model consists of McNealtype electrical cable models of myelinated nerve fibers, representing DC fibers and DR fibers. All nodal membranes are made excitable using the equations by Chiu et al., transformed to a temperature of $37^{\circ} \mathrm{C}$. The rostrocaudal DC fibers issue transverse collaterals into the dorsal horns. DR fibers bifurcate into an ascending and a descending DC fiber upon entering the spinal cord, whereas their orientation in the surrounding cerebrospinal fluid can be varied. The potentials in the $3 \mathrm{D}$ model at the nodal positions of DC and DR fibers are used to calculate the threshold voltages for their excitation when applying a rectangular voltage pulse $(0.21 \mathrm{~ms})[1,3]$. 


\section{RESULTS}

Two different approaches have been used to improve the stimulation of DC fibers, thereby reducing the activation of DR fibers. In one approach the geometry of the conventional longitudinal anode-cathode array was optimized, taking advantage of the different orientations of $D C$ and DR fibers. In the second approach a transversely placed array was used, taking advantage of both the different positions and orientations of the two fiber types.

\section{Longitudinal Electrode Array}

Monopoles and longitudinal bi/tripoles were placed in the dorsal epidural space of volume conductor models at symmetrical positions to the spinal cord [4]. Simulations have shown that the threshold stimulus to excite a (longitudinal) DC fiber is reduced as compared to the threshold stimulus of a (transverse) DR fiber, when:

- a bipole is used instead of a monopole (cathode);

- a tripole (central cathode) is used instead of a bipole;

- spacing of anodal and cathodal contacts is reduced;

rostro-caudal contact size is reduced;

- lateral contact size is reduced.

The preferential stimulation of DC fibers is maximized by a combination of these factors: tripolar stimulation with small-sized and narrowly separated contacts. This results in a high longitudinal driving function (2nd derivative of the longitudinal field potential) in the dorsal columns. In contrast, monopolar stimulation with a long cathode favors DR fiber activation. In this situation the main dircction of the current near the center of the cathode is in a transverse plane, thus imposing the highest driving function along DR fibers near their entry in the spinal cord.

The threshold stimulus ratio of DC and DR fibers is also affected by an anatomical parameter: the distance between the epidural electrode and the spinal cord, which varies along the vertebral column. The DC fiber threshold rises steeper than the DR fiber threshold as this distance is increased [4]. Therefore, DC fibers can easily be activated when stimulating at a cervical level, but usually not at a midthoracic level.

\section{Transverse Tripolar Array}

A second method to suppress the activation of DR fibers is by using a transverse tripole with a central cathode and anodes on both sides. The tripole was placed symmetrically in the dorsal epidural space of $3 \mathrm{D}$ models. The lateral anodes were placed near the dorsal roots, thus "shielding" DR fibers for their excitation. Simulations have shown that DR fibers and lateral DC fibers have increased thresholds in comparison to fibers in the median dorsal columns [5]. This method, based on fiber-position selective stimulation, can be improved when a contact geometry, favoring fiber orientation selectivity, is used. Therefore, the lateral anodes should be long, to maximize "shielding" of DR fibers, whereas the central cathode should be small to minimize the threshold stimulus for DC fiber activation.

In addition to the "shielding" of DR fibers, the transverse electrode arrangement can also be used to "steer" the electrical field in the dorsal columns in order to activate those DC fibers corresponding to the painful dermatomes. Therefore, the transverse tripole should be powered by a dual channel stimulator, giving simultaneous constant voltage pulses with separate amplitude control. When the pulse amplitude of the right channel is reduced, while keeping the amplitude of the left channel constant, the right anode will increasingly behave like a cathode, whereas the current density near the left anode will increase. As a result, the area of activated DC fibers will shift to the right side of the dorsal columns. This "steering" capability enables the correction of insufficient paresthesia coverage (i.e., after electrode dislocation) without surgical intervention.

\section{CONCLUSIONS}

SCS electrodes meant to give improved dorsal column stimulation, needed for the management of complex chronic pain, were designed by computer modeling. Two electrodes are proposed: a longitudinal tripole (central cathode) having small contact size and spacing, and a transverse tripole (central cathode). The latter also enables a change of the activated dorsal column area by varying the output amplitude ratio of a dual channel stimulator. Clinical evaluation of the new methods is in progress. Preliminary results are in accordance with model predictions.

Acknowledgements. The investigations were supported by a grant from Medtronic, Inc., Minneapolis, Minnesota.

\section{REFERENCES}

[1] J.J. Struijk, J. Holsheimer, H.B.K. Boom, "Excitation of dorsal root fibers in spinal cord stimulation: a theoretical study", IEEE Trans. Biomed. Eng., vol. 40, pp. 632-639, 1993.

[2] J.J. Struijk, J. Holsheimer, G. Barolat, J. He, H.B.K. Boom, "Paresthesia thresholds in spinal cord stimulation: a comparison of thcoretical results with clinical data", IEEE Trans. Rehab. Eng., vol. 1, pp. 101-108, 1993.

[3] J.J. Struijk, J. Holsheimer, G.G. van der Heide, H.B.K. Boom, "Recruitment of dorsal column fibers in spinal cord stimulation: influence of collateral branching", IEEE Trans. Biomed. Eng., vol. 39, pp. 903-912, 1992.

[4] J. Holsheimer, J.J. Struijk, N.R. Tas, "Effects of electrode geometry and combination on nerve fibre selectivity in spinal cord stimulation", Med. Biol. Eng. Comp., vol. 33, pp. 676-682, 1995.

[5] J.J. Struijk, J. Holsheimer, "Transverse tripolar spinal cord stimulation: theoretical performance of a dual channel system", Med. Biol. Eng. Comp., vol. 34 (in press). 